\title{
Cross-reactivity between sera from dogs experimentally infected with Dirofilaria immitis and crude extract of Toxocara canis
}

\author{
Kun-Ho SONG ${ }^{1)}$, Mineo HAYASAKI ${ }^{2)}$, Kyu-Woan $\mathrm{CHO}^{1)}$, \\ Sang-Eun $\mathrm{LEE}^{1)}$ and Duck-Hwan $\mathrm{KIM}^{1) *}$ \\ ${ }^{1)}$ Laboratory of Veterinary Internal Medicine, College of Veterinary Medicine, Chungnam National \\ University, Daejeon 305-764, Korea, ${ }^{2}$ Veterinary Clinical Center, School of Veterinary Medicine, \\ Yamaguchi University, Yamaguchi 753-8515, Japan
}

\begin{abstract}
This study was performed to investigate whethere there is cross-reactivity between Dirofilaria immitis and three intestinal nematodes of dogs. In ELISA, D. immitisinfected dog sera obtained at the 4 th molting stage (9-11 weeks) and microfilaremic stage (25-30 weeks) were shown to be highly reactive with crude extract of $T$. canis. In immunoblotting, some antigenic fractions, 44,57,88, $100 \mathrm{kDa}$ of crude extract of $T$. canis, were found to be positive reaction with sera of dogs infected with $D$. immitis. However, little or no cross-reaction were observed between sera of $D$. immitis-infected dogs and crude extract antigen of $T$. vulpis or A. caninum. These result suggest that there are partial cross reaction between sera of $D$. immitis-infected dogs and the antigen of $T$. canis.

Key words: Dirofilaria immitis, cross-reactivity, ELISA, immunoblotting, Toxocara canis
\end{abstract}

Employing the indirect hemagglutination assay (Hayasaki, 1981) and Dot-ELISA (Matsumura et al., 1984), the authors found no cross-reactivity was observed between Dirofilaria immitis and intestinal nematodes including $T$. canis. On the other hand, Ott et al. (1985) reported the presence of immunologically distinguishable speciesspecific non-cross reactive antigens and the presence of cross-reactive antigens in antigenic extracts obtained from adult $D$. immitis and $T$. canis. Additionally, Konno et al. (1995) reported that D. immitis possessed a partial antigenic cross reactivity to $T$. canis, revealed by using

\footnotetext{
- Received 24 October 2002, accepted after revision 27 November 2002.

*Corresponding author (e-mail: dhkim@cnu. ac.kr)
}

immunoblotting. The above consideration indicate that more strict analysis is required for the study of antigenic cross-reactivity between D. immitis and other parasites. This study was performed to examine on cross-reactivity between $D$. immitis and three intestinal nematodes of dogs.

Five healthy mongrel juvenile dogs, approximately three months of age, were housed in mosquito-proof runs and treated with piperazine $(100 \mathrm{mg} / \mathrm{kg}, \mathrm{PO})$ for intestinal parasites prior to experimentation. Three dogs were experimentally infected with $D$. immitis by the procedures described by Hayasaki (1982). Briefly, mosquitoes, Aedes togoi, collected during the larval stage from their natural spawning areas were reared under laboratory conditions and inoculated by feeding on a dog with about 200 circulating microfilarial (Mf) counts per $20 \mu \mathrm{l}$ of blood. Two dogs were used 
as the negative control. Infective larvae (L3) of $D$. immitis were recovered from the proboscis of the infected mosquitoes 10 to 14 days after blood feeding and suspended in saline during microscopic observation. Three dogs were subcutaneously injected in the inguinal region with 228 (dog No. 1), 278 (dog No. 2) and 248 (dog No. 3) infective larvae (L3). The blood was collected from the cephalic vein once a week, and the sera were stored at $-70^{\circ} \mathrm{C}$ until analysis. The crude extract antigens of $T$. canis, T. vulpis and A. caninum were prepared as previously described (Hayasaki, 1982). Briefly, the crude antigens used in this study were extracted from adult worms of $T$. canis, $A$. caninum and T. vulpis by phosphate buffered saline (PBS, pH 7.2, 0.1 M). These worms were homogenized and sonicated by tissue homogenizer $\left(15 \mathrm{~min}, 4^{\circ} \mathrm{C}\right)$ and ultrasonicator (50 watt, $15 \mathrm{~min}, 4^{\circ} \mathrm{C}$ ), respectively, and then allowed to incubate overnight at $4^{\circ} \mathrm{C}$. After centrifugation at $18,000 \mathrm{~g}$, the supernatant as antigen was collected and kept at $-70^{\circ} \mathrm{C}$. The protein concentration of the antigens was determined using the methods of Lowry et al. (1951). ELISA was performed by method described by Grieve et al. (1981). SDS-PAGE and immunoblotting was performed as described by Gonno and Hayasaki (1995). In this study, by ELISA, D. immitis-infected sera obtained at the 4th molting stage (9-11 weeks after infection) and microfilaremic stage (25-30 weeks after infection) were strongly reacted with crude extract of $T$. canis. However, little or no cross-reactivities were found between $D$. immitis-infected sera and crude extracts of $T$. vulpis or A. caninum.

Immunoblotting analysis are more effective application of immunologic diagnosis (Kaneko et al., 1990; Hayasaki et al., 1994). In this study, 44, 57, 88 and $100 \mathrm{kDa}$, which were protein bands of crude extract of $T$. canis, were shown to be strongly reactive with $D$. immitisinfected sera. Based on these findings, we suggest that $D$. immitis share a common antigen with $T$. canis. This cross-reactivity may be dependent on many antigenic epitopes existing in somatic components of the parasites because previous report indicate that $D$. immitis consists of many protein components and its antigenicity is very complex, and also these two parasites may be originated from a same progenitor and still possess partially similar antigenicity. Therefore, certain interspecies antigenic epitopes may be isolated from one species which may be useful in eliciting a highly specific immune response in a host toward cross-reactive epitopes in different species (Hayasaki et al., 1994).

Further studies are needed to elucidate the

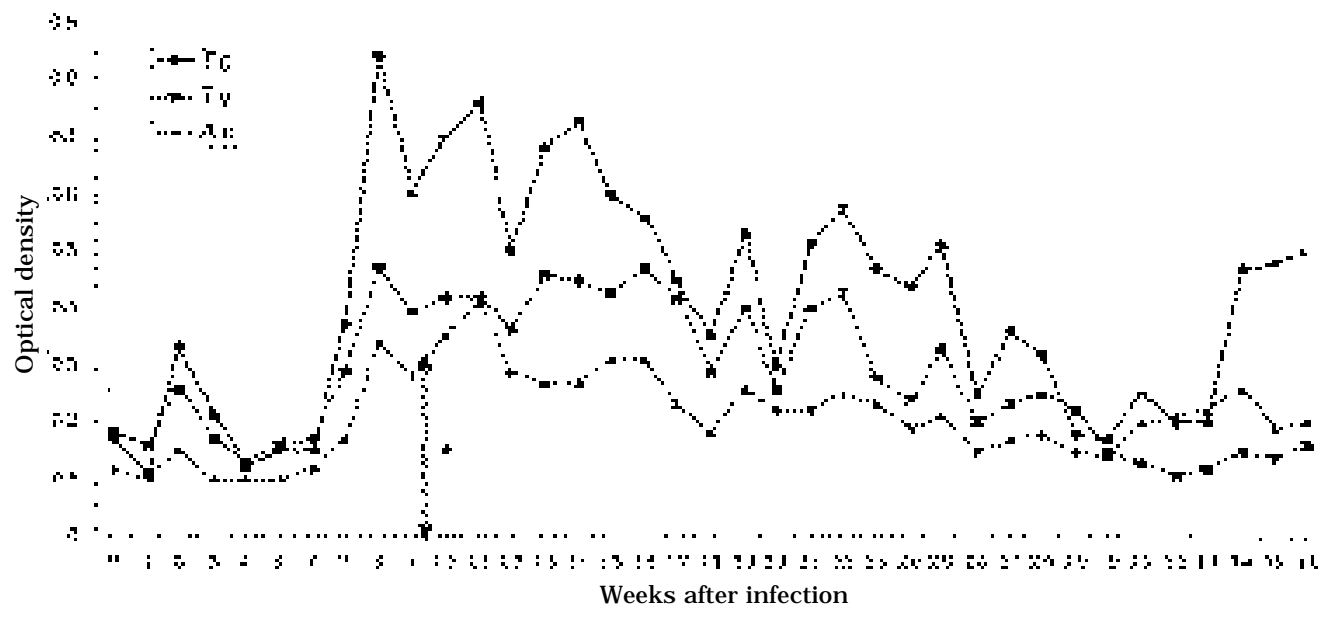

Tc: Toxocara canis Tv: Trichuris vulpis Ac: Anclostoma caninum

Fig. 1. Antibody levels of $D$. immitis-infected dog serum (No. 1) against crude extract antigens of three intestinal nematodes, measured by ELISA. Asterisk represents the range of optical density of control group. 


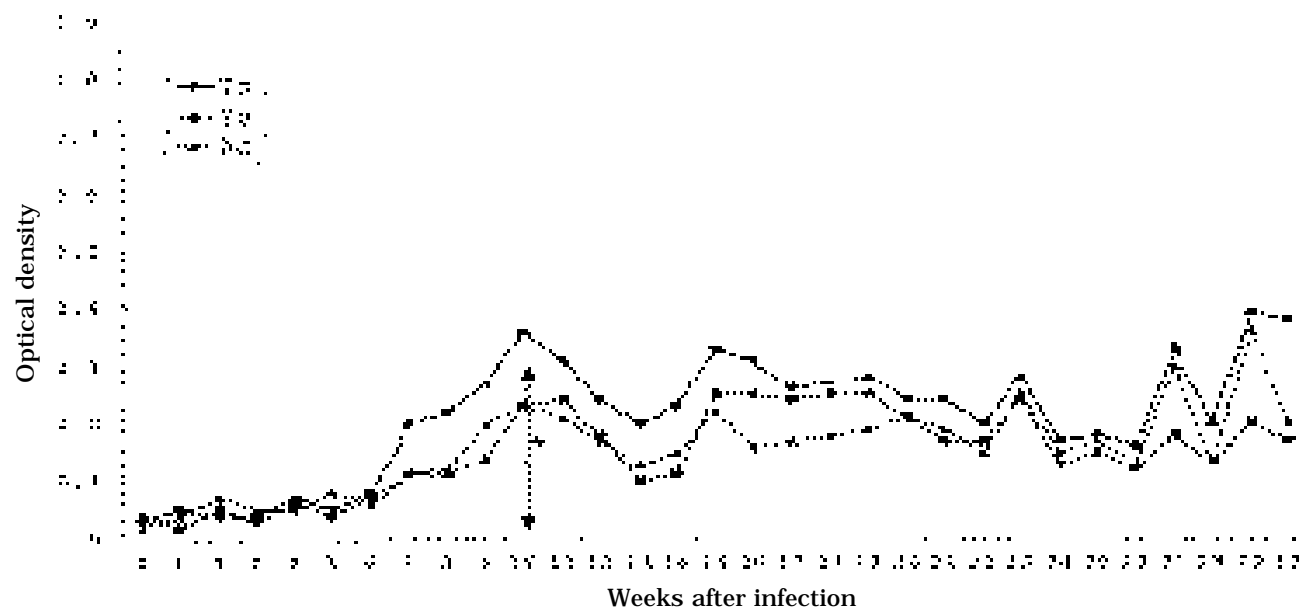

Tc: Toxocara canis Tv: Trichuris vulpis Ac: Anclostoma caninum

Fig. 2. Antibody levels of $D$. immitis-infected dog serum (No. 2) against crude extract antigens of three intestinal nematodes, measured by ELISA. Asterisk represents the range of optical density of control group.

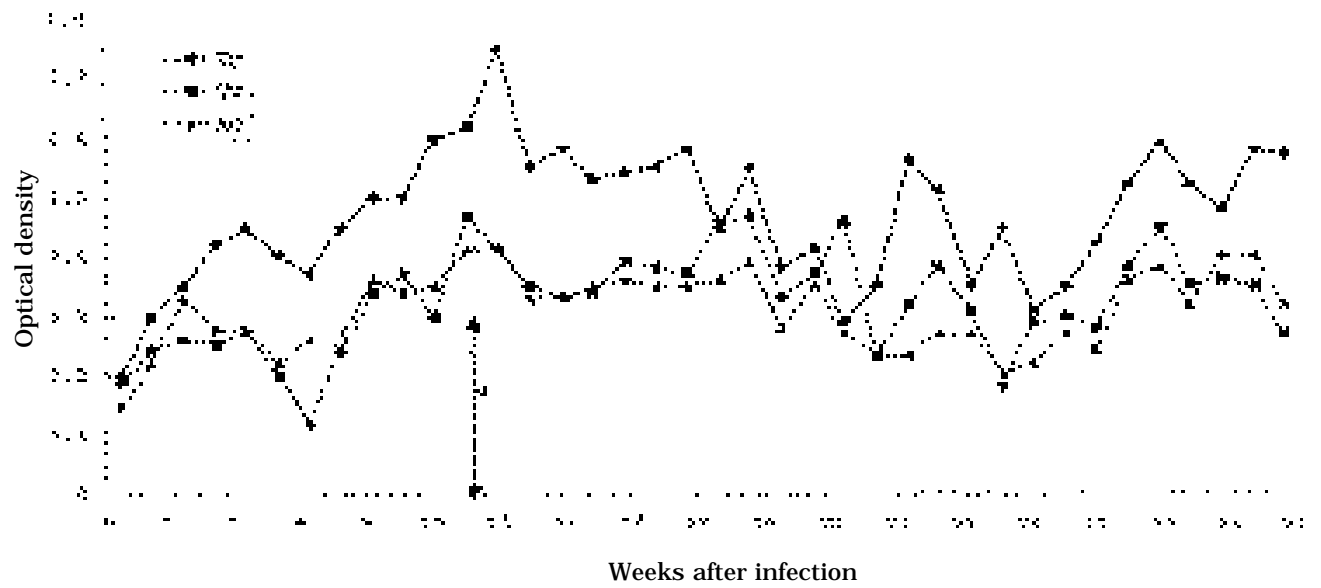

Tc: Toxocara canis Tv: Trichuris vulpis Ac: Anclostoma caninum

Fig. 3. Antibody levels of $D$. immitis-infected dog serum (No. 1) against crude extract antigens of three intestinal nematodes, measured by ELISA. Asterisk represents the range of optical density of control group.

immunological implications antigenic crossreactivity between $D$. immitis and intestinal nematodes including $T$. canis.

\section{REFERENCES}

Grieve RB, Johnson MM, Jacobson RH (1981)

Enzyme-linked immunosorbent assay for measurement of antibody responses to Dirofilaria immitis in experimentally infected dogs. Am $J$ Vet Res 42: 66-69.

Hayasaki M (1981) Indirect hemmaglutination test for diagnosis of canine filariasis. Jpn $J$ Vet Sci 43: 21-26.

Hayasaki M (1982) Reaginic and hemmaglutinating antibody production in dogs infected 


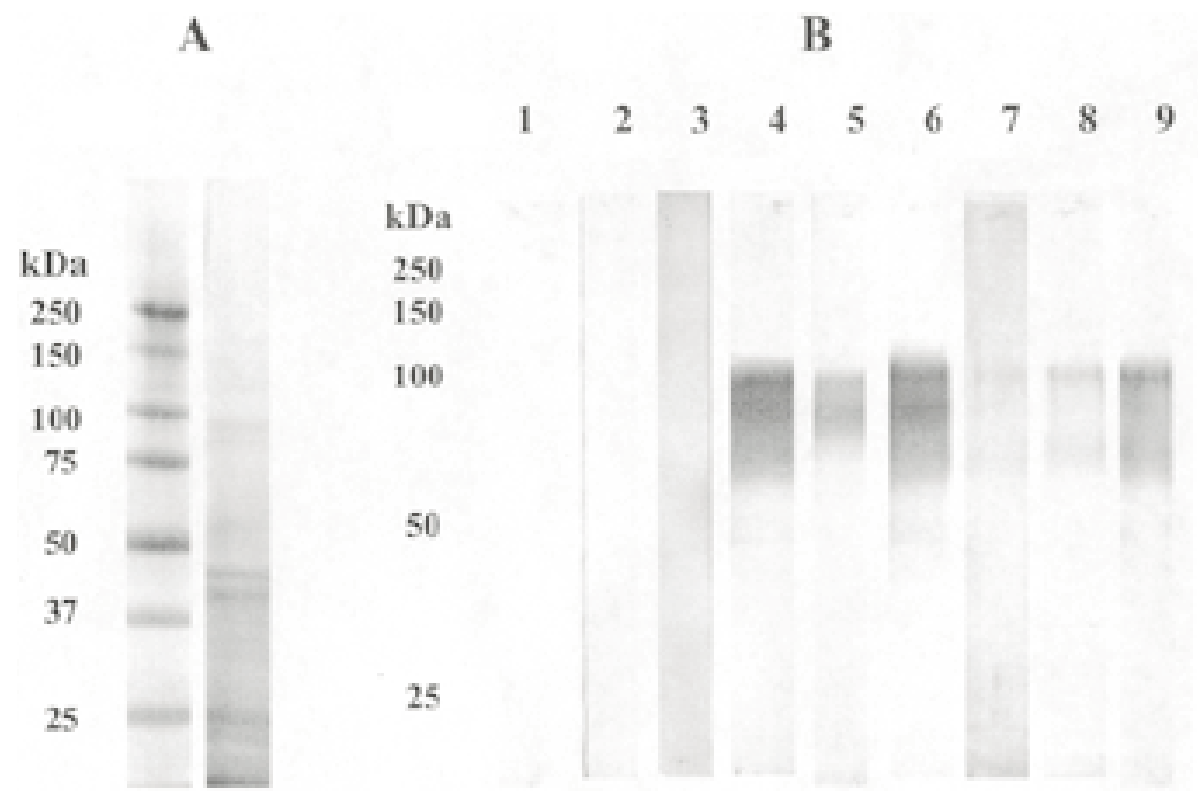

Fig. 4. Protein fractions of adult $T$. canis were separated by SDS-PAGE stained with Coomassie brilliant blue R-250 (A), and antigenic cross reactions between $T$. canis antigens and sera from dogs experimentally infected with $D$. immitis by immunoblotting (B). Lane 1-3: uninfected serum, lane 4-6: sera collected at the period of prepatent infection, lane 7-9: sera collected at the period of patent infection (lane 1, 4, 7: $\operatorname{dog} 1$, land 2, 5, 8: $\operatorname{dog} 2$, lane 3, 6, 9: $\operatorname{dog} 3$ ).

with Dirofilaria immitis. Jpn J Vet Sci 44: 6370.

Hayasaki M, Nakamura F, Konno K(1994) Immunoblotting analysis of somatic components of Dirofilaria immitis. J Vet Med Sci 56: 1181-1183.

Kaneko H, Hayasaki M, Ohishi I (1990) Antigenic identification of excretory-secretory products of adult Dirofilaria immitis. Jpn $J$ Vet Sci 52: 995-1000.

Konno K, Hayasaki M (1995) Antigenic cross reactivity among Dirofilaria immitis and the four intestinal parasite-species in the dog. Jpn J Parasitol 44: 161-164.
Lowry OH, Rosenbrough NJ, Farr AL, Randall RJ (1951) Protein measurement with the folin phenol reagent. J Biol Chem 193: 265-275.

Matsumura K, Kazuta Y, Endo R, Tanaka K (1984) Detection of circulating toxocaral antigens in dogs by sandwich enzyme-immunoassay. Immunology 51: 609-613.

Ott RA, Staples M, Weekley M, Maggio ET (1985) Demonstration of both immunogenically unique and common antigenic determinants in Dirofilaria immitis and Toxocara canis using monoclonal antibodies. Vet Immunol Immunopathol 10: 147-153. 